\title{
Direct digital synthesis and current mirror based software defined general purpose visible light communication transmitter
}

\author{
Tore Leikanger and Juha Häkkinen \\ Circuits and Systems (CAS) \\ Univeristy of Oulu \\ Oulu, Finland \\ tore.leikanger@oulu.fi
}

\begin{abstract}
In this paper, a visible light communication transmitter using a LED matrix with low-cost LEDs is shown. This design use a DAC to control the LED drivers, through a discrete component current mirror circuit. The DAC is controlled using an FPGA, which together works as a highly configurable direct digital synthesiser. The design proposed in this paper is thus well suited as a transmitter node in a software defined radio using visible light communication.

In this paper, we describe the design, and show its behaviour when programmed to transmit a number of carriers modulated with amplitude and phase modulation schemes.

Index Terms-Direct Digital Synthesis, DDS, Visible Light Communication, VLC, Transmitter, Light-Emitting Diode, LED, Software Defined Radio, SDR
\end{abstract}

\section{INTRODUCTION}

The use of light-emitting diodes (LED) is an increasingly popular choice for a lighting sources [1]. Furthermore, LEDs have been shown to feature other properties, for example that the switching speed is higher than other lighting technologies. This property makes LEDs an excellent choice for a wireless data communication channel. [2]

Several modulation techniques are used in visible light communicaions (VLC) technologies [3]. Many approaches, however, use specific circuitry for the modulation, as is done by B. Lin et.al. [4], for example.

Modern communication designs are often made as software defined radios (SDR), where the control of the modulation is moved from the hardware to the software. These require general purpose communication hardware, capable of several modulation schemes. [5]

In this paper, we will look into a general purpose VLC transmitter (TX) node design, using direct digital synthesis (DDS) and discrete component current mirrors. This approach enables the proposed transmitter design to be used as a modulation scheme independent SDR transmitter node. We will first introduce the design of the transmitter. Then, by using an ACcoupled photodiode receiver circuit and an oscilloscope, we will show how this circuit perform in different situations. The paper is then concluded by a discussion about the approach and results.

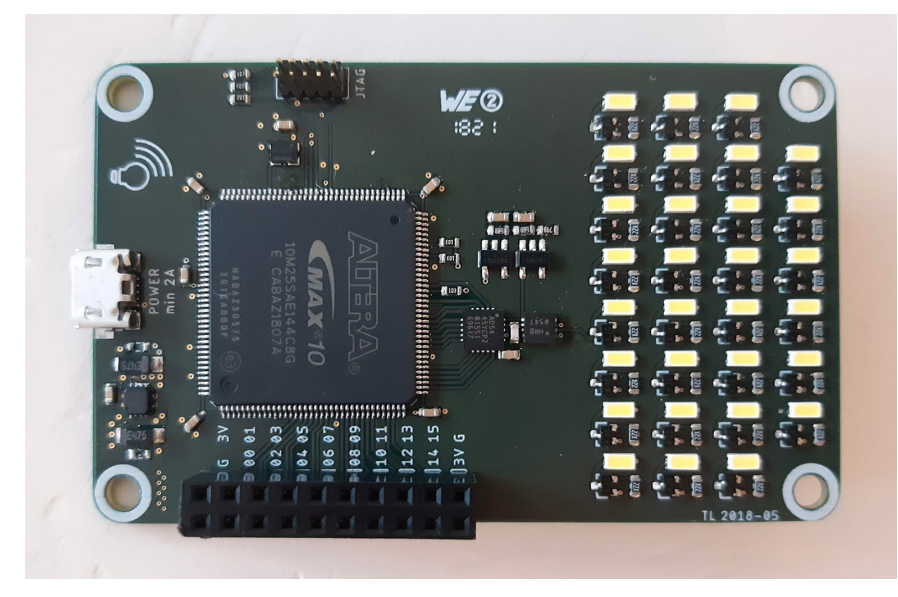

Fig. 1. Picture of the VLC DDS TX node.

\section{DESIGN}

The developed VLC transmitter, shown in fig. 1, is designed around an field-programmable gate array (FPGA) and a highspeed digital-to-analog converter (DAC) with 20 MSPS. An array of 30 low-cost Luxeon 3014 white LEDs is used as the lighting element, and thus also the VLC transmitter. Each LED is giving about $24 \mathrm{~lm}$ at $60 \mathrm{~mA}$, resulting in a total luminous flux of about $720 \mathrm{~lm}$ [6].

The current through the LEDs are controlled directly by the DAC, using a trans-conductance amplifier shown in fig. 2. The driver circuit of a single LED is used as the reference driver, from which the current is copied to the other LED drivers. A degenerated current mirror is used while copying the current, as can be seen in the schematics in fig. 2. Because the current mirrors are degenerated, the reference driver work as a current source, while the rest of the drivers need the same degenerating resistance to make the current as equal to the reference as possible.

The current mirror is sensitive to the design uncertainties of the transistors, especially with discrete transistors when layout techniques to minimise these errors are not possible to use. 


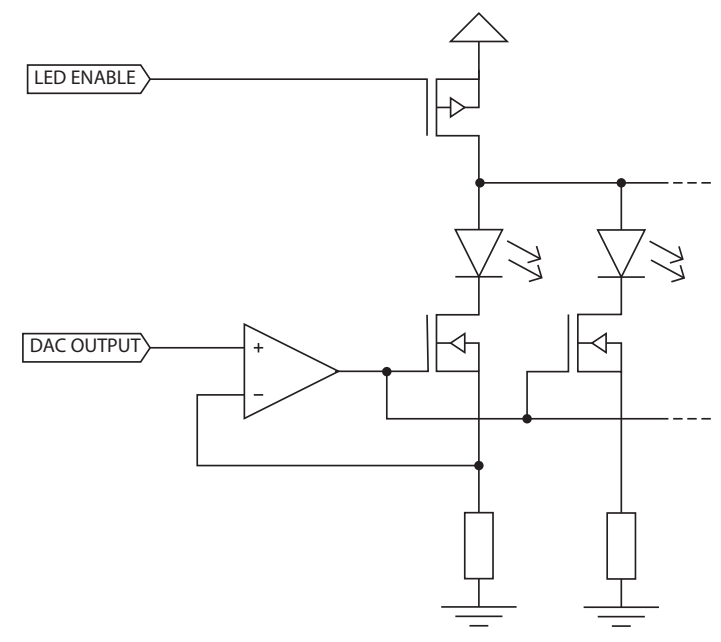

Fig. 2. The LED driver and current mirror circuit, with LED enable switch shown. The reference driver is shown, together with a single slave driver.

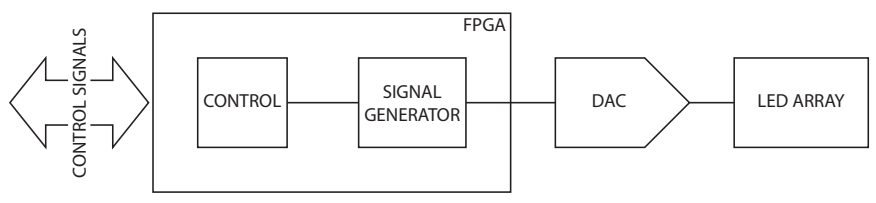

Fig. 3. Block diagram of the circuit.

However, we are working with the sum of the light intensity from all LEDs in the matrix, and the precision of the light intensity of each individual LED is thus not of importance. Moreover, the communication is in the modulation of the light, and not the accurate light intensity. Using inaccurate current mirrors to drive the LEDs is thus an efficient and well suited technique for this purpose.

The DAC is driven by the FPGA, which together makes an direct digital synthesiser (DDS), as can be seen in the block diagram in fig. 3. Using an FPGA and a DAC to design the DDS makes it highly configurable, which in turn makes this system feasible as a software-defined radio (SDR) transmitter node. The proposed design can change from being a simple on-off keying (OOK) transmitter without a carrier to a complex multi-carrier orthogonal frequency-division multiplexing (OFDM) transmitter, for example. Also, because no AC-coupling happens between the FPGA and the driver circuits, fading of the light can be easily adjusted with the DC-level of the signal.

Now, to test the functionality of the system, an AC-coupled photo diode (PD) receiver circuit connected to an oscilloscope is used. The transmitter circuit as well as the receiver circuit are mounted inside a rack with a distance of about $15 \mathrm{~cm}$. This set-up can be seen in fig. 4 .

\section{Measurements}

The performance of the circuit was measured by using a simple PD and trans-impedance amplifier (TIA) circuit, probed with an oscilloscope. This circuit can be seen in fig. 5 .

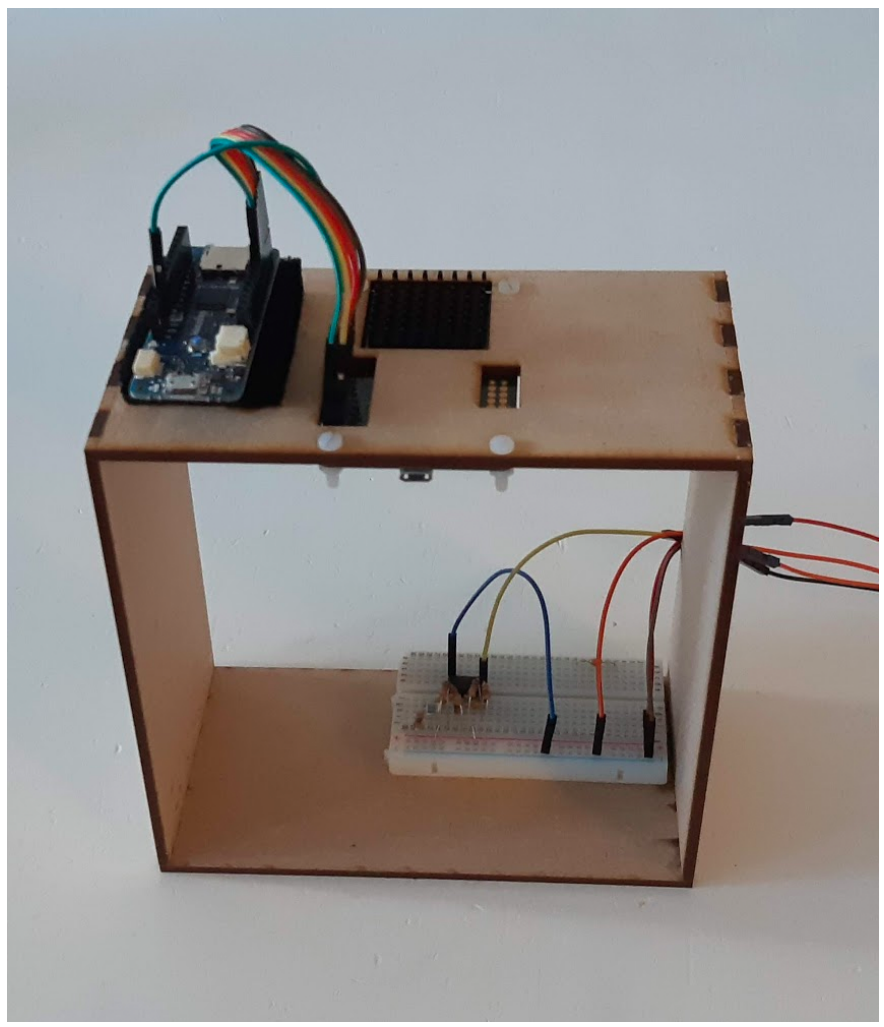

Fig. 4. The measurement set-up. The VLC TX node is below the roof of the rack, where only the cooling ribbon, the control lines, and the programming port is visible from the top. A VLC receiver analog front-end circuit is on the floor of the rack, and a microcontroller circuit that controls the VLC TX node is on the top of the rack.

To perform the measurements, the FPGA was configured to generate a carrier with frequency as controlled by an external micro-controller (MCU) circuit, and modulated with either amplitude modulation (AM) or phase modulation (PM). In addition, the design enables the MCU to control the LED enable switch shown in fig. 2, as well as to enable and disable the carrier signal.

The performance of the carrier is measured by generating carrier signals of different frequencies, and to look at the timedomain signals as well as the frequency domain spectrums, as can be seen in fig. 6. We further used the design to generate a waveform to measure the performance of the DDS during different modulation schemes as well. This is shown in fig. 7, where a $1 \mathrm{MHz}$ carrier signal is enabled for about $30 \mu \mathrm{s}$, during which about $10 \mu$ s are modulated with AM and PM modulations of different magnitudes.

The measurements of the carrier signals show a carrier signal peak at about $-20 \mathrm{~dB}$ relative to the DC peak, as can be seen in fig. 6. This amplitude is kept close to the same level between $200 \mathrm{kHz}$ and $1 \mathrm{MHz}$, meaning close to no attenuation has happened in this range. Harmonics are apparent at about $-60 \mathrm{~dB}$, and the noise floor is at about $-80 \mathrm{~dB}$, i.e. about 40 $\mathrm{dB}$ and $60 \mathrm{~dB}$ lower signal than the carrier. The sinusoidal generated by the DDS, transmitted by the LED array, and received by the receiver is thus a fairly clean signal with no 


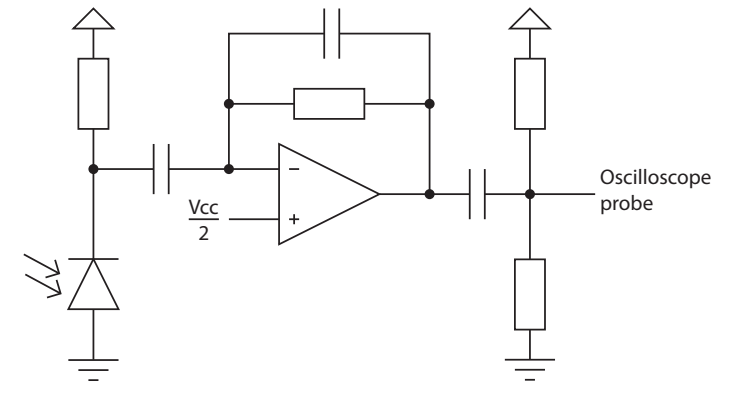

Fig. 5. The VLC receiver analog front-end used in the measurements.

significant noise.

As can be seen in fig. 7, this approach is capable of modulating the carrier in a number of ways. Here, we have modulated the carrier using amplitude- and phase modulations, with different modulation strengths. Also, an AM modulated dual-carrier signal is shown. The number of carriers can be increased to feature multiple carriers with more advanced
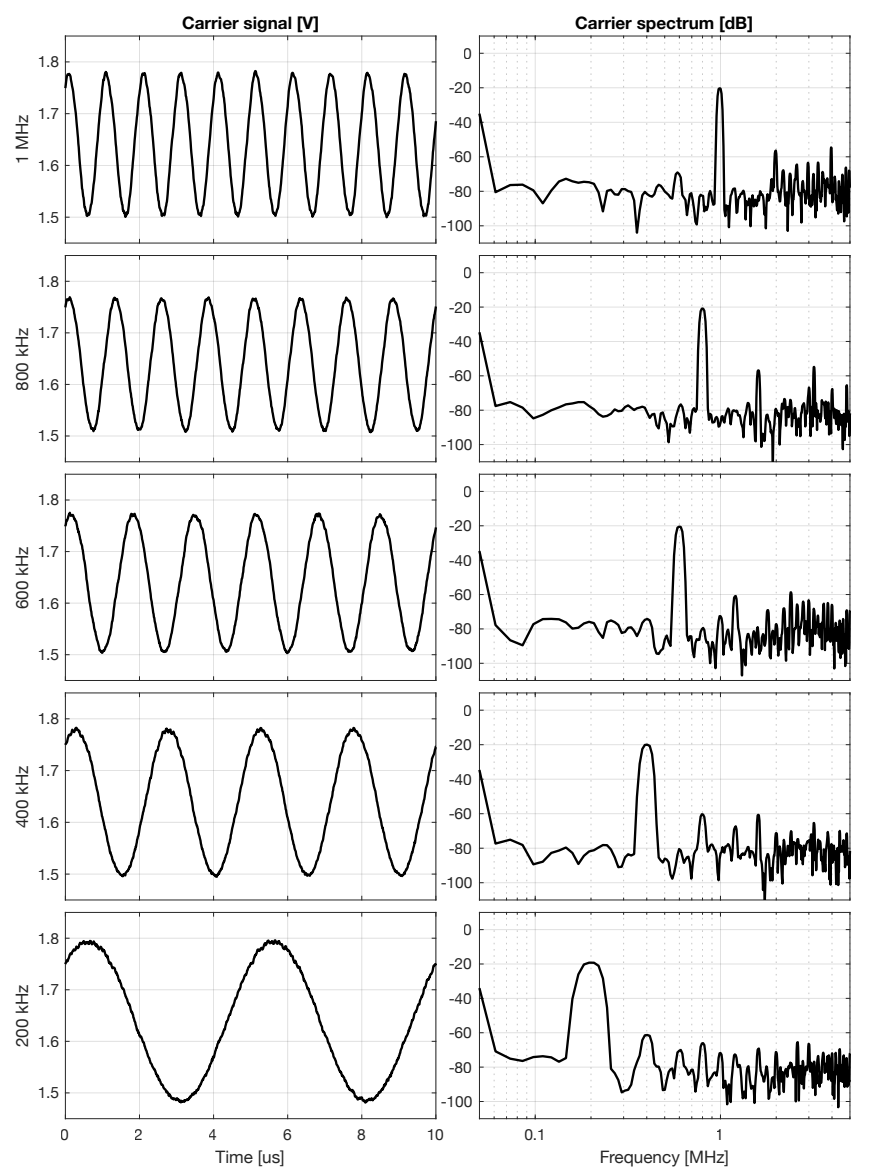

Fig. 6. Measurements of the carrier signal generated by the VLC DDS TX node in the range of $200 \mathrm{kHz}$ to $1 \mathrm{MHz}$. The carrier signal in time domain is shown to the left, and its spectrum is shown to the right. The unit of the $y$-axis are volts for time domain signal and decibel for the frequency domain spectrum.

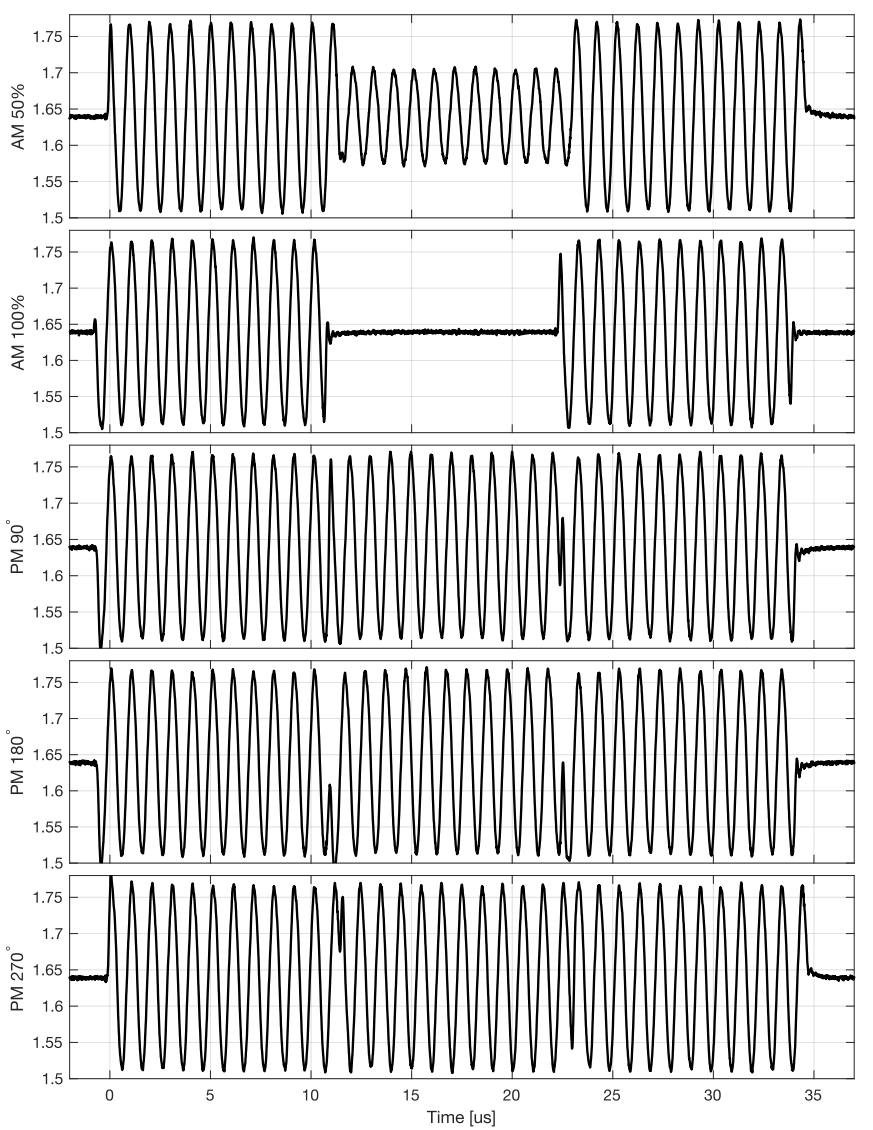

Fig. 7. The received signal as measured on the oscilloscope, when a single 1 $\mathrm{MHz}$ carrier signal is turned on for about $30 \mu \mathrm{s}$. The carrier is unmodulated for about $10 \mu \mathrm{s}$, modulated with different modulation schemes for about 10 $\mu \mathrm{s}$, and then again unmodulated for about $10 \mu \mathrm{s}$ before the carrier is turned off.
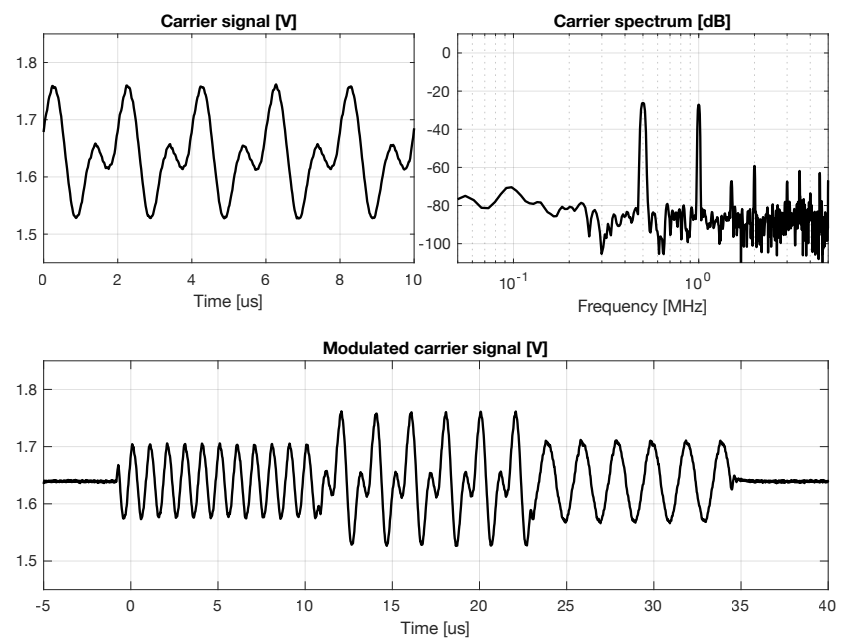

Fig. 8. Carrier measurement with spectrum, and modulation behaviour of a dual carrier signal with $100 \%$ AM modulation. The carriers are orthogonal, with frequencies of $500 \mathrm{kHz}$ and $1 \mathrm{MHz}$. The carrier modulation sequence is as follows: Both modulated, $500 \mathrm{kHz}$ carrier modulated, none modulated, 1 $\mathrm{MHz}$ carrier modulated, both modulated. 
modulation schemes. Also, in fig. 8, a dual carrier scheme is shown, and how this behaves when the carriers are modulated independently with $100 \%$ AM modulation.

\section{DISCUSSION}

Using a carrier-based data transmission in VLC communication has an advantage over using flickering-based data transmission, because the luminous intensity of the light is easier to keep at the same level during data transmission. This is possible to achieve using the proposed approach to design a general purpose VLC transmitter node. Furthermore, we have shown that a reasonably clean sinusoidal carrier can be generated and transmitted through the designed LED array. The proposed design enables the carrier to be modulated using several modulation techniques, and even include several modulated carriers in the same transmission. The proposed approach can thus handle both simple carrier-less or single carrier modulations as well as complex multiple-carrier modulation techniques.

However, to improve the quality of the sinusoidal, we would need a faster DAC. A faster DAC would also increase the maximum frequency of the carrier that can be generated using the proposed approach, and thus enable faster data transmission rates.

\section{CONCLUSION}

We have seen that the proposed design gives a working solution for a software defined radio using visible light. The proposed approach, using discrete current mirrors to control the LED matrix drivers has been shown to give good control over the transmitter LEDs, while at the same time enable analog control over the generated light intensity. This provides both DC and AC control over the LEDs, and thus both fading and modulation of the LEDs can be done through the DAC. The carriers generated with the design was shown in both time- and frequency domain, as seen from a photodiode receiver connected to an oscilloscope through a trans-impedance amplifier circuit. Carriers with frequencies up to $1 \mathrm{MHz}$, and with different modulations was shown. Furhter a dual-carrier example with AM modulation was shown, where frequencies of $500 \mathrm{kHz}$ and $1 \mathrm{MHz}$ was used.

Also, improvements of the design was discussed. Increasing the speed of the DAC, and thus the driver circuit, was argued to be the most critical and beneficial improvement of the circuit.

\section{REFERENCES}

[1] M. Wendt and J. Andriesse, "Leds in real lighting applications: from niche markets to general lighting," in Conference Record of the 2006 IEEE Industry Applications Conference Forty-First IAS Annual Meeting, vol. 5, Oct 2006, pp. 2601-2603.

[2] S. Singh, G. Kakamanshadi, and S. Gupta, "Visible light communicationan emerging wireless communication technology," in 2015 2nd International Conference on Recent Advances in Engineering Computational Sciences (RAECS), Dec 2015, pp. 1-3.

[3] E. Shinwasusin, C. Charoenlarpnopparut, P. Suksompong, and A. Taparugssanagorn, "Modulation performance for visible light communications," in 2015 6th International Conference of Information and Communication Technology for Embedded Systems (IC-ICTES), March 2015, pp. 1-4.
[4] B. Lin, X. Tang, Z. Ghassemlooy, C. Lin, M. Zhang, Z. Zhou, Y. Wu, and $\mathrm{H}$. Li, "A noma scheme for visible light communications using a single carrier transmission," in 2017 First South American Colloquium on Visible Light Communications (SACVLC), Nov 2017, pp. 1-4.

[5] A. C. Tribble, "The software defined radio: Fact and fiction," in 2008 IEEE Radio and Wireless Symposium, Jan 2008, pp. 5-8.

[6] Lumileds, "Luxeon 3014,” Luxeon 3014 datasheet, 2017. 